\title{
Which ultrasound transducer type is best for diagnosing pneumothorax?
}

\author{
R. Ketelaars ${ }^{1,2^{*}} \mathbb{D}$, E. Gülpinar ${ }^{1}$, T. Roes ${ }^{1}$, M. Kuut ${ }^{1}$ and G. J. van Geffen ${ }^{1,2}$
}

\begin{abstract}
Background: An accurate physical examination is essential in the care of critically ill and injured patients. However, to diagnose or exclude a pneumothorax, chest auscultation is unreliable compared to lung ultrasonography. In the dynamic prehospital environment, it is desirable to have the best possible ultrasound transducer readily available. The objective is to assess the difference between a linear-array, curved-array, and phased-array ultrasound transducer in the assessment for pneumothorax and to determine which is best.

Methods: In this double-blinded, cross-sectional, observational study, 15 observers, experienced in lung ultrasonography, each assessed 66 blinded ultrasound video clips of either normal ventilation or pneumothorax that were recorded with three types of ultrasound transducers. The clips were recorded in 11 adult patients that underwent thoracoscopic lung surgery immediately before and after the surgeon opened the thorax. The diagnostic accuracy of the three transducers, elapsed time until a diagnosis was made, and the perceived image quality was recorded.

Results: In total, 15 observers assessed 990 ultrasound video clips. The overall sensitivity and specificity were 98.2\% and $97.2 \%$, relatively. No significant difference was found in the diagnostic performance between transducers. A diagnosis was made slightly faster in the linear-array transducer clips, compared to the phased-array transducer $(p=.031)$. For the linear-, curved-, and phased-array transducer, the image quality was rated at a median (interquartile range $[\mathrm{IQR}])$ of 4 (IQR 3-4), $3(\mathrm{IQR} 2-4)$, and 2 (IQR 1-2), relatively. Between the transducers, the difference in image quality was significant $(p<.0001)$.
\end{abstract}

Conclusions: There was no difference in diagnostic performance of the three transducers. Based on image quality, the linear-array transducer might be preferred for (prehospital) lung ultrasonography for the diagnosis of pneumothorax.

Keywords: Ultrasonography, Transducer, Pneumothorax, Emergency medical services

\section{Background}

In the critically ill and injured patient, an accurate physical examination is essential in the care of the patient. However, auscultating for breath sounds in a respiratory distressed patient is often difficult or even impossible, especially in a noisy accident scene, or patient compartment of a (moving) ambulance or helicopter.

The sensitivity of auscultation for the diagnosis of hemothorax, hemopneumothorax, and pneumothorax

\footnotetext{
*Correspondence: Rein.Ketelaars@radboudumc.nl

${ }^{1}$ Department of Anesthesiology, Pain and Palliative Medicine, Radboud University Medical Center, Radboud Institute for Health Sciences, Geert Grooteplein-Zuid 10,6525 GA Nijmegen, The Netherlands

Full list of author information is available at the end of the article
}

is only $58-66 \%$ [1-3]. Unilateral decreased or absent breath sounds are often interpreted as a pneumothorax. However, conditions such as splinting from rib pain, lung contusion, atelectasis, pneumonia, pleural effusion, and tumor growth may account for the same abnormal auscultation.

Lung ultrasonography (US) for the diagnosis of pneumothorax was first described in 1986 [4]. It may rule-in pneumothorax with a sensitivity ranging from 81 to $98 \%$ and rule it out with a specificity approaching $99-100 \%$ [5-7]. Additionally, pleural effusion, lung contusion, and atelectasis may be detected [7]. It has even been suggested that US might 1 day replace the stethoscope $[8,9]$. 
US is feasible in the prehospital setting including inside ground ambulances and a helicopter emergency medical service (HEMS) [10-12]. Similar to most diagnostic and therapeutic procedures, US requires training and regular practice. Time pressure and limited working space are additional challenges [12]. To facilitate the best possible images, it is important that optimally set-up US equipment is readily available. In an optimal configuration, the most suitable transducer is connected to the US machine.

Lung US can be performed with high-frequency lineararray, curved-array, or phased-array transducers. However, it is not known which one is preferable and provides the best images.

We hypothesized that a linear-array transducer is the optimal transducer for the appreciation of the pleural line for diagnosing pneumothorax. The aim of the study is to compare three transducer types on diagnostic accuracy, speed of the diagnosis, and image quality in the assessment for pneumothorax.

\section{Methods}

We performed a double-blinded, cross-sectional, observational study to compare three types of ultrasound transducers for the diagnosis of two conditions: normal ventilation, and pneumothorax. Ethical approval was obtained from the institutional ethics review board of the Radboud university medical center, Nijmegen. Written informed consent was asked and obtained from every patient and from every observer.

At the preoperative outpatient evaluation clinic of the Radboud university medical center, Nijmegen, the Netherlands, from September to October 2017, we recruited a consecutive series of eleven eligible patients that were scheduled for video-assisted thoracoscopic surgery (VATS) for pulmonary, mainly neoplastic, pathology. The inclusion criteria were a body mass index $<30 \mathrm{~kg} \mathrm{~m}^{-2}$ and the absence of pathology of the chest wall, visceral or parietal pleura.

Lung US is a valuable test for the detection or exclusion of a pneumothorax [13, 14]. A US transducer is positioned on the chest wall perpendicular to two adjacent ribs. Between the acoustic shadows of two ribs, a hyperechoic line is visible representing the interface of the parietal and visceral pleura. With normal ventilation, lung sliding is observed as a to-and-fro movement at the pleural line as a result of the sliding of the visceral pleura against the inner chest wall. B-lines may be observed as hyperechoic lines radiating down from the pleural line. Their presence excludes pneumothorax (at the transducer position). Horizontal repetitions of the pleural line appearing below at multiples of the skin-pleural line distance are called A-lines. Their appearance is more prominent in the presence of a pneumothorax when $\mathrm{B}$-lines are absent and no longer obscuring the A-lines.

We used a portable X-Porte ultrasound system (Fujifilm SonoSite Inc., Bothell, WA, USA) equipped with three transducers: a high-frequency linear-array $15-6 \mathrm{MHz}$ (HFL50xp), a curved-array abdominal 5-2 MHz (C60xp) and a phased-array cardiac 5-1 MHz (P21xp) transducer. The footprints of the transducers are $5 \mathrm{~cm}, 6 \mathrm{~cm}$, and $2.1 \mathrm{~cm}$, respectively.

For the VATS procedure, isolated ventilation of the dependent lung via a double-lumen endotracheal tube was necessary. First, all patients underwent general anesthesia, were intubated and ventilated, and placed in a lateral decubitus position. The ventilator was set to deliver a tidal volume of $5 \mathrm{ml} \mathrm{kg}^{-1}$ at a rate of $20 \mathrm{~min}^{-1}$. The anesthesiologist verified the position and depth of the doublelumen endotracheal tube with fiberoptic bronchoscopy.

Second, the linear array, curved-array, and phasedarray transducer were positioned over the fourth or fifth intercostal space at the axillary line in a craniocaudal orientation. 15-second ultrasound video clips were recorded of normal ventilation at a respiratory rate of $20 \mathrm{~min}^{-1}$. The zone of interest was the pleural line with its two adjacent ribs. A typical clip was framed as shown in Fig. 1.

Third, after the chest was prepped and draped, ventilation of the non-dependent lung was interrupted while the surgeon opened the chest, introduced the videoscope and visually confirmed the collapse of the lung. Thereafter, the surgeon recorded three similar 15-s video clips of the established pneumothorax with the three transducers wrapped in sterile transducer covers (Fig. 2). Hence, six clips were recorded in every patient. The time interval between the induction of the pneumothorax (reference test) and the performance of the three ultrasound

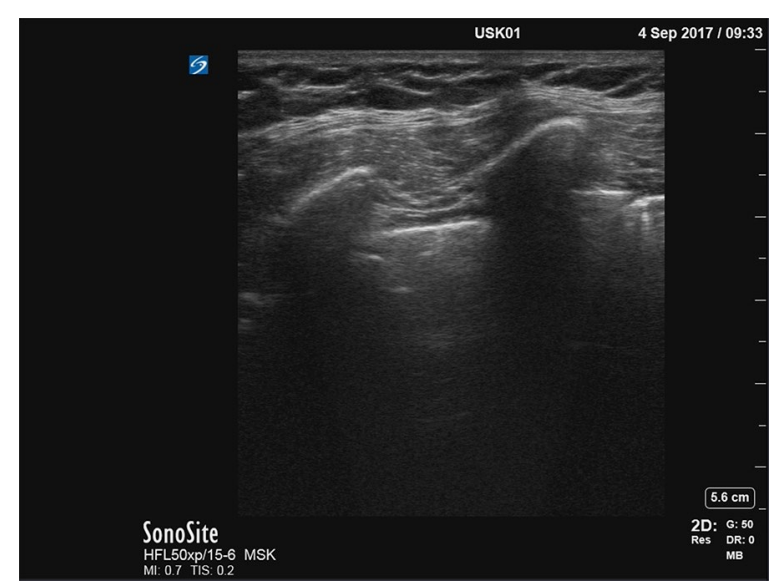

Fig. 1 A typical uncropped image of the pleural interface, acquired with the phased-array transducer. On the right, the cropped version is displayed as is was played back to the observers 


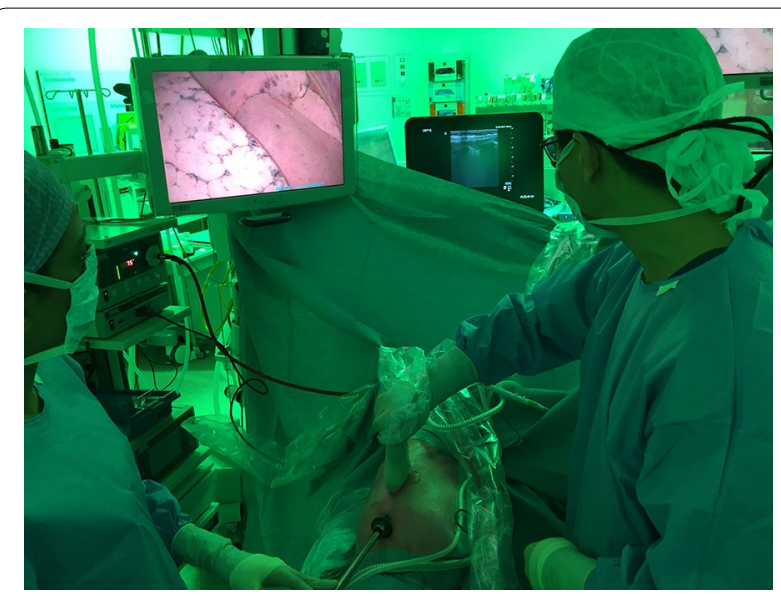

Fig. 2 The surgeon performs lung ultrasonography in a patient with a confirmed pneumothorax and the videoscope in situ. The video screen displays an image of the inside of the right hemi-thorax and the collapsed right lung. The surgeon is handling the wrapped-up ultrasound transducer. The ultrasound device is shown in the back of the image

video clips was no longer than $2 \mathrm{~min}$. No adverse events occurred.

We cropped the video clips using iMovie for OS X, version 10.1.8 (Apple Inc., Cupertino, CA, USA). After we cropped and removed the text from the captured video clips, it was now no longer possible for the observers and the researchers to reliably recognize the transducer type by the image shape (rectangular or sector-shaped). An uncropped still image of the video clip and its cropped version are displayed in Fig. 3.

The Nijmegen physician-staffed HEMS carries a portable US machine since 2006. All HEMS physicians were trained in lung US either at the introduction of the US machine or at the start of their employment. They use lung US regularly in their prehospital practice.

We recruited all 13 HEMS physicians (except the author, RK) and two anesthesiology residents with extensive experience in lung ultrasonography as observers to assess a randomized set of 66 15-s ultrasound clips. We used PotPlayer for Windows, version 1.7 (Kakao Corp., Jeju, South Korea) to separately randomize and playback the cropped set of clips for each observer (Fig. 4). Before the observers assessed the set of video clips, they were informed about how we acquired the clips and about the two possible conditions (normal ventilation and pneumothorax). Due to the cropping and randomization, the observers were blinded for the diagnosis and for the transducer type.

The observers were requested to pause the playback themselves when they were certain about the diagnosis based on the presence or lack of lung sliding, A-line sign, or B-lines. The equally blinded researcher recorded the elapsed time (s), the observer's diagnosis and perceived image quality rated on a 1-5 Likert scale (1, very poor; 5 , very good). For every observer, their experience (years) with lung US and preferred transducer type for lung US was recorded.

\section{Statistical analysis}

Normally distributed data are reported as mean and standard deviation (SD). Data with an asymmetrical distribution are reported as median and interquartile range (IQR). We calculated the difference in elapsed time and image quality between transducers in every patient and observer: linear-array vs curved-array; linear-array vs phased-array; curved-array vs phased-array. Then, we

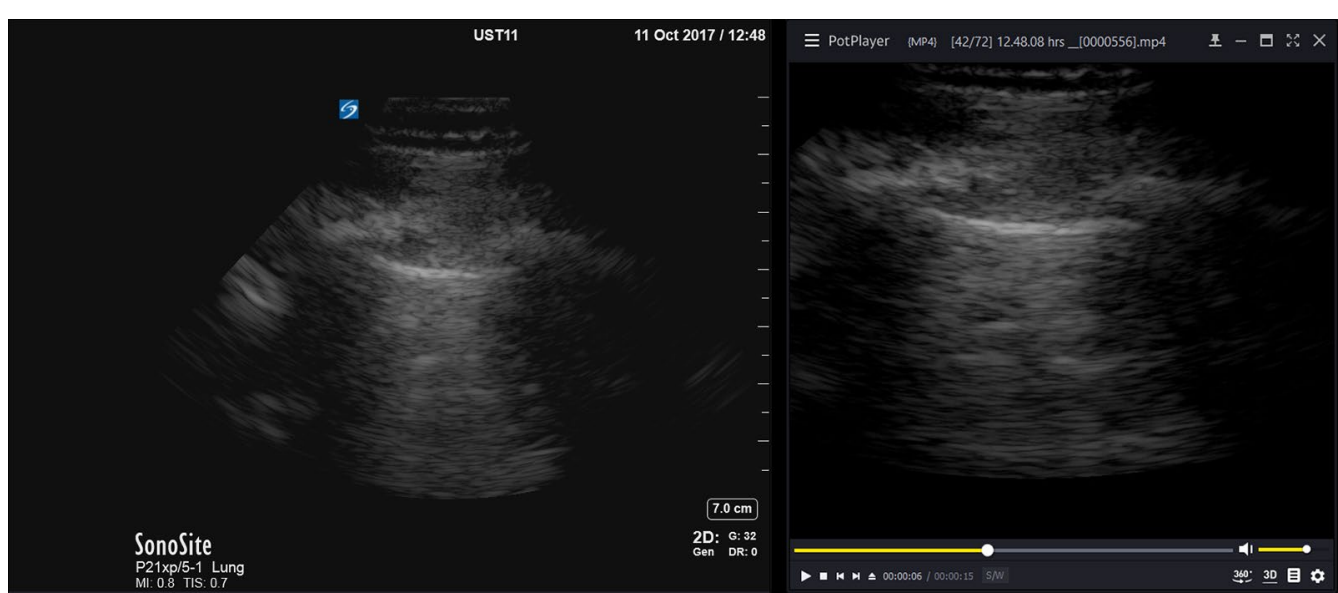

Fig. 3 A typical uncropped and cropped image of the pleural interface, acquired with the phased-array transducer. On the right, the cropped version is displayed as it was played back to the observers 


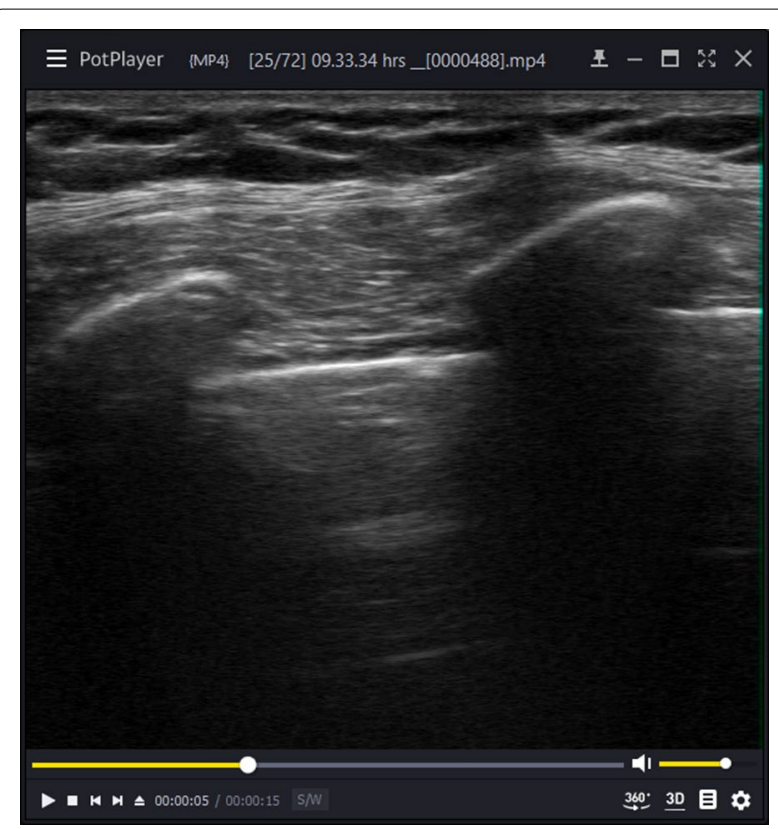

Fig. 4 A cropped 15-s clip played in a random order to the observers

fitted a linear mixed model with a random intercept to these differences to account for clustering within each observer. A two-tailed McNemar's test for clustered data (Durkalski's Chi-square test) was used to test for differences between the transducers in diagnostic performance. The Wilcoxon signed-rank test was used to test for differences in time until final diagnosis between diagnoses (normal ventilation and pneumothorax). For all statistical tests, significance level was set to .05. For statistical analysis, IBM SPSS Statistics for Windows, version 25.0 (IBM Corp., Armonk, NY, USA) and R, version 3.4.1, lme4 package installed (R Foundation for Statistical Computing, Vienna, Austria) were used.

\section{Results}

\section{Patients}

Sixty-six lung US video clips were acquired in 11 patients, of whom eight women, with a mean age of 64.0 years $( \pm 9.03)$. Their mean weight was $66.1 \mathrm{~kg}( \pm 9.30)$ and the mean body mass index (BMI) was $24.3 \mathrm{~kg} \mathrm{~m}^{-2}( \pm 2.98)$. Surgery was performed on the left and right chest in four and seven cases, respectively. All participants had a pneumothorax after the surgeon opened the thorax.

\section{Observers}

The video clips were observed by 15 physicians of whom 13 HEMS physicians (nine anesthesiologists, and four trauma surgeons) and two anesthesiology residents. These observers all had extensive experience (a mean of 7.1 years $[ \pm 3.58])$ in lung ultrasonography.
Prior to the observations, six observers indicated to prefer a linear-array transducer for lung ultrasonography. Seven preferred a phased-array transducer and two had no preference. The curved-array transducer was preferred by none.

Each observer assessed the 66 cropped clips in a random order. There was no significant difference between their different backgrounds for success rate (correct or incorrect diagnosis) or time they needed to assess the video clips.

In 10 of the 990 judged clips, an observer could not decide on the diagnosis because the image quality was perceived to be too bad. Therefore, for data analysis where the diagnosis is a factor we used the data on 980 clips. The time was recorded from the start of video playback to the moment the observer declared to be unable to state a diagnose.

\section{Diagnostic performance}

The overall sensitivity and specificity was $98.2 \%$ and 97.2\%, positive predictive value (PPV) and negative predictive value (NPV) was $97.2 \%$ and $98.2 \%$, respectively. A cross tabulation of the correct and incorrect diagnosis compared between transducers are displayed in Table 1.

The diagnostic performance measures for the different transducers for pneumothorax were calculated for 980 assessed clips and are displayed in Table 2.

McNemar's test for clustered data showed no significant difference in diagnostic performance between the different transducers. (Linear- vs curved-array: $p=.706$, linear- vs phased-array: $p=.537$, curved- vs phasedarray: $p=.515)$.

\section{Time}

The time the observers needed to reach a diagnosis is displayed in Table 3 and Fig. 5.

After we fitted the linear mixed model, we found a significant difference between the times that elapsed until a final diagnosis was made. Whit the linear-array transducer the diagnosis was made $.51 \mathrm{~s}(p=.031)$ faster compared with the phased-array transducer. The curvedarray transducer was $.15 \mathrm{~s}(p=.049)$ faster than the phased-array transducer. We found no significant difference between the linear- and curved-array transducers. These comparisons between transducers are displayed in Table 4.

Normal ventilation was diagnosed significantly faster than the diagnosis of a pneumothorax, regardless of transducer type. The Wilcoxon signed-rank test showed a significant difference overall $(p<.0001)$ and within the three transducer groups as shown in Fig. $5(p<.0001$ in all three groups). 
Table 1 Cross tabulation of the number of correct and incorrect diagnoses compared between transducers

\begin{tabular}{|c|c|c|c|c|}
\hline & Diagnosis & Correct & Incorrect & Tota \\
\hline & & Curved-array transducer & & \\
\hline \multirow[t]{2}{*}{ Linear-array transducer } & Correct & 313 & 7 & 320 \\
\hline & Incorrect & 8 & 2 & 10 \\
\hline \multirow[t]{2}{*}{ Total } & & 321 & 9 & 330 \\
\hline & & Phased-array transducer & & \\
\hline \multirow[t]{2}{*}{ Linear-array transducer } & Correct & 308 & 12 & 320 \\
\hline & Incorrect & 8 & 2 & 10 \\
\hline \multirow[t]{2}{*}{ Total } & & 316 & 14 & 330 \\
\hline & & Phased-array transducer & & \\
\hline \multirow[t]{2}{*}{ Curved-array transducer } & Correct & 309 & 12 & 321 \\
\hline & Incorrect & 7 & 2 & 9 \\
\hline Total & & 316 & 14 & 330 \\
\hline
\end{tabular}

Table 2 Diagnostic performance of the three ultrasound transducers for pneumothorax

\begin{tabular}{lllll}
\hline & $\begin{array}{l}\text { Linear-array } \\
\text { transducer } \\
(\%)\end{array}$ & $\begin{array}{l}\text { Curved- } \\
\text { array } \\
\text { transducer } \\
(\%)\end{array}$ & $\begin{array}{l}\text { Phased- } \\
\text { array } \\
\text { transducer } \\
(\%)\end{array}$ & $\begin{array}{l}\text { All } \\
\text { transducers } \\
\text { combined } \\
(\%)\end{array}$ \\
\hline Sensitivity & 97.5 & 98.2 & 98.8 & 98.2 \\
Specificity & 97.6 & 96.4 & 97.5 & 97.2 \\
\hline
\end{tabular}

Table 3 Time elapsed until a final diagnosis was made

\begin{tabular}{lllll}
\hline Diagnosis & $\begin{array}{l}\text { Linear- } \\
\text { array } \\
\text { transducer }\end{array}$ & $\begin{array}{l}\text { Curved- } \\
\text { array } \\
\text { transducer }\end{array}$ & $\begin{array}{l}\text { Phased- } \\
\text { array } \\
\text { transducer }\end{array}$ & $\begin{array}{l}\text { All } \\
\text { transducers } \\
\text { combined }\end{array}$ \\
\hline $\begin{array}{l}\text { Normal ven- } \\
\text { tilation }\end{array}$ & $2(1-5)$ & $3(1-5.5)$ & $3(2-5.5)$ & $3(1-5)$ \\
$\begin{array}{c}\text { Pneumotho- } \\
\text { rax }\end{array}$ & $5(3-7)$ & $5(3-7)$ & $6(3.5-8.5)$ & $5(3-7)$ \\
$\begin{array}{c}\text { All } \\
\text { diagnoses }\end{array}$ & $4(2-6)$ & $4(2-6)$ & $4(2-7)$ & $4(2-6.25)$ \\
\hline
\end{tabular}

The data are presented as median seconds (interquartile range)

a This includes the ten clips without diagnosis

\section{Image quality}

Image quality was scored on a 5-point Likert scale. The image quality of the linear-, curved-, and phased-array transducers was appreciated at a median of 4 (IQR 3-4), 3 (IQR 2-4); 2 (IQR 1-2), respectively. Overall image quality was rated a median of 3 (IQR 2-4). The distribution of the image quality scores per transducer type is displayed in Fig. 6.

The Wilcoxon signed-rank test showed a significant difference in image quality between all three transducers $(p<.0001)$.
After we fitted the linear mixed model, we found significant differences in image quality between all three transducers. The image quality with the linear-array transducer was 1.78 higher than the image quality with the phased-array transducer on a 5-point Likert scale. These comparisons in image quality between transducers are displayed in Table 5.

The image quality was deemed too bad to make a diagnosis in ten cases: 8 of 330 phased-array transducer clips and 2 of 330 linear-array transducer clips. Of those, seven clips showed a pneumothorax and three showed normal lung sliding.

\section{Discussion}

In this study, we found no difference in the diagnostic performance of the linear-array, curved-array, and phased-array transducer. The diagnostic performance was very good: sensitivity, specificity, PPV, and NPV were all between 96.4 and $98.8 \%$. The observers needed an additional $.51 \mathrm{~s}$ to reach a final diagnosis with the phasedarray transducer compared to the linear-array transducer. A final diagnosis was reached much faster when lung sliding was present regardless of the transducer type.

The image quality scored by the observers on a 1-5 scale was significantly different between all three transducers; the linear-array transducer achieved the best scores, the phased-array transducer the worst. Moreover, the image quality was too bad to reach a diagnosis in eight phased-array transducer clips and two linear-array transducer clips.

These findings suggest that the actual diagnostic performance of the three transducers for pneumothorax is comparable. However, these experienced observers perceived the best image quality and needed the 


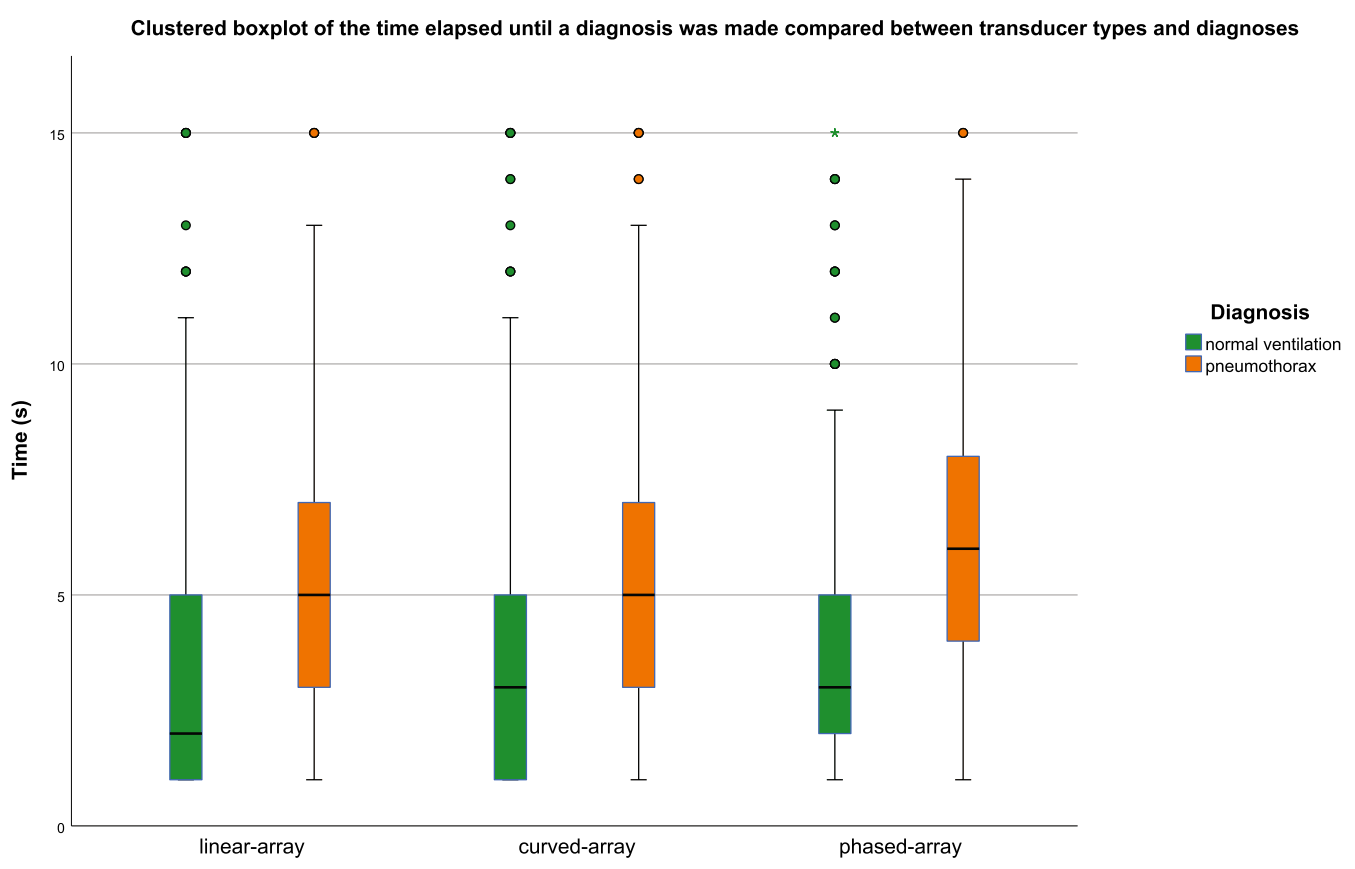

Fig. 5 Boxplot of the elapsed time until a diagnosis was made compared between transducer types and diagnoses. The elapsed time until a diagnosis was stated by the observers. The time is represented in median seconds. The box represents the $25-75 \%$ interquartile range. The whiskers indicate the $95 \%$ confidence interval. There is a significant difference in the elapsed time until a diagnosis was made between normal ventilation and pneumothorax within all three transducers $(p<.0001)$

Table 4 Difference in time elapsed until a diagnosis was made between transducer types

\begin{tabular}{llc}
\hline Compared transducers & Estimate $[\mathbf{9 5 \%} \mathrm{Cl}]$ & $\boldsymbol{p}$ value \\
\hline Linear-array vs curved-array & $-.35[-.78, .07]$ & .105 \\
Linear-array vs phased-array & $-.51[-.97,-.05]$ & .031 \\
Curved-array vs phased-array & $-.15[-.59, .28]$ & .049 \\
\hline
\end{tabular}

This table presents the differences in elapsed time until a diagnosis was made between a combination of two transducers, using a linear mixed model with a random intercept

The differences are presented in seconds

A negative value indicates that less time elapsed using the left of the two compared transducers

least amount of time when they judged the lineararray transducer clips. Based on these findings, the linear-array transducer might qualify as the preferred transducer for lung ultrasonography. However, the transducer choice may depend on more important factors such as the intended gamut of indications US is used for and whether the machine will be equipped with one or more transducers. In a single transducer setup, the best choice is probably a curved-array or a phased-array transducer to be able to evaluate both the abdomen and pericardium, in addition to the chest.
To our knowledge, there are no studies that have compared US transducers for diagnosing pneumothorax in a similar study design.

In a study with a comparable design, but not focused on pneumothorax, the authors compared a $10-5 \mathrm{MHz}$ and a $14-5 \mathrm{MHz}$ linear-array transducer for a wide array of emergency department point-of-care ultrasound indications [15]. However, lung ultrasonography was discussed only briefly. Overall, their observers most frequently preferred the $10-5 \mathrm{MHz}$ transducer over the $14-5 \mathrm{MHz}$ transducer.

In another study, the investigators compared a 5-10 $\mathrm{MHz}$ linear-array and a $1-5 \mathrm{MHz}$ phasedarray sector transducer in 55 patients scheduled for a thoracic-computed tomography (CT) scan [16]. The authors evaluated the diagnostic performance for pneumothorax, pleural effusion, consolidation, and interstitial syndrome. In six patients with a pneumothorax, confirmed with $\mathrm{CT}$, they found that the linear-array transducer performed best with a sensitivity and specificity of $83 \%$ and $100 \%$, respectively. The phased-array transducer showed a sensitivity and specificity of $67 \%$ and $100 \%$. Sensitivity of both auscultation and chest radiography was only $50 \%$. In our study, the gold standard was a thoracoscopically induced and confirmed pneumothorax. Because we assessed 495 ultrasound 


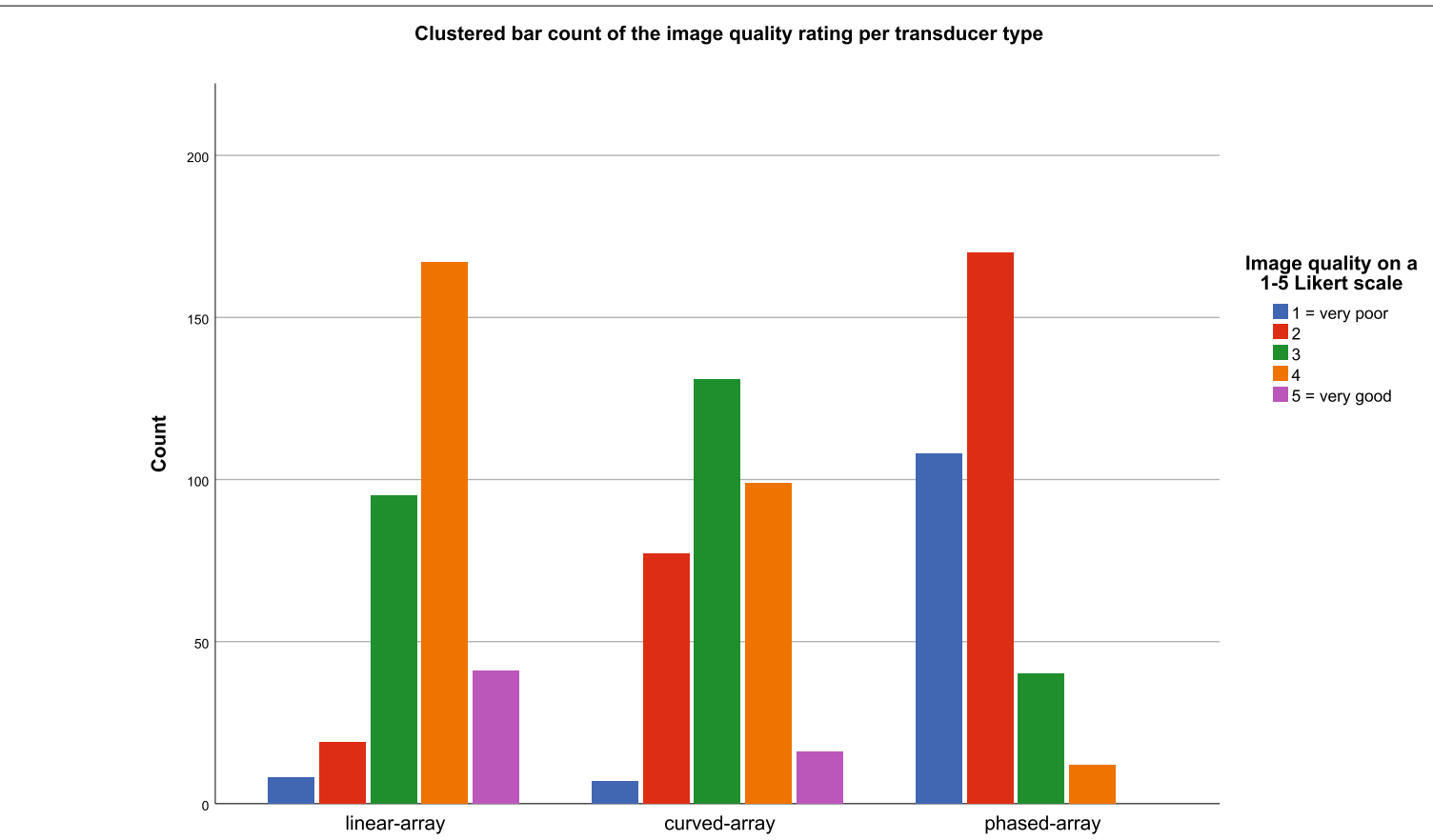

Fig. 6 Clustered bar count of the image quality rating per transducer type. The Wilcoxon signed-rank test showed a significant difference in image quality between all three transducers $(p<.0001)$

Table 5 Difference in image quality between transducer types

\begin{tabular}{lcc}
\hline Compared transducers & Estimate $[\mathbf{9 5 \%} \mathrm{Cl}]$ & $\boldsymbol{p}$ value \\
\hline Linear-array vs curved-array & $.53[.29, .76]$ & $<.0001$ \\
Linear-array vs phased-array & $1.78[1.56,2.01]$ & $<.0001$ \\
Curved-array vs phased-array & $1.25[1.09,1.42]$ & $<.0001$ \\
\hline
\end{tabular}

This table presents the differences in reported image quality between a combination of two transducers, using a linear mixed model with a random intercept

The image quality was reported on a 5-point Likert scale: 1 , very poor; 5 , very good

A positive value indicates that the image quality was better with the left of the two compared transducers

clips showing pneumothorax, the diagnostic performance we found is more reliable.

We hypothesized that the linear-array transducer would have the best diagnostic performance. This study, however, showed no difference in diagnostic accuracy between the transducers.

The Nijmegen HEMS introduced prehospital ultrasonography to the Netherlands in 2006 and used a phased-array transducer ever since. Only years later, a linear-array transducer was added. A curved-array transducer has never been used. This history might explain the transducer preferences of the observers and the high and equal diagnostic performance between transducers.
Although diagnostic performance is equal, we recommend the linear-array transducer for (prehospital) lung ultrasonography. The diagnosis is made faster and with a better image quality. These are important advantages in the dynamic prehospital environment, HEMS physicians encounter challenges such as time pressure, limited working space, residual clothing, defibrillator pads, and Velcro ${ }^{\circledR}$ straps. Most importantly, the interpretation of US images may be hampered by sunlight or precipitation. When the HEMS physicians have the best possible image quality, they can better deal with these factors and do the best possible for our patients.

Furthermore, when the linear-array transducer is installed as the default transducer, it may have additional advantages. It is the preferred transducer for vascular access and assessment of the airway and endotracheal tube position [15]. These matters often take precedence over detailed assessment of breathing, although it may be of vital importance to be informed about a significant pneumothorax before airway management is commenced.

In addition, the linear-array transducer is superior for ultrasound-guided regional anesthesia (UGRA) in severely injured or trapped extremities and for optic nerve sheath diameter (ONSD) measurements in traumatic brain injury (TBI) patients [17]. For abdominal ultrasound and echocardiography, however, the phasedarray or curved-array transducer is still invaluable. 
The observers were able to successfully assess the video clips of normal ventilation and pneumothorax without having access to the US machine or the patient. This situation is comparable to a telemedicine setup in which the US operator could be at a different physical location than the observer of the images. Therefore, we agree that lung ultrasound can be successfully used in telemedicine setups [18].

\section{Strengths and limitations}

We chose a unique approach to select VATS patients with a freshly induced and visually confirmed pneumothorax as the gold standard. In addition, we included the video clips of the same patients with normal anatomy before surgery. Another unique aspect was the cropping of the video clips thus transducers could not be identified by any text or image or sector shape.

A limitation of this study is that it might be underpowered because we could not demonstrate a difference in diagnostic performance between transducers. It could also mean the difference is close to none.

Another limitation is that we informed the observers that all patients were ventilated similarly and that besides a pneumothorax in half of the video clips, no other pathology was present. This could be an advantage for them judging the clips and might have resulted in an overestimation of the diagnostic performance and time needed. The performance could have been even better when we acquired M-mode clips looking for lung pulse or clips that included the lung point [14].

Conversely, most observers were uncomfortable with the fact that they had to assess video clips and that they were therefore unable to reposition or adjust the transducer, adjust the gain or depth, or compare with the contralateral chest. In addition, it was regarded a disadvantage that no additional clinical parameters were provided. The setting of pulmonary surgery introduced some minor challenges. In some clips, lung sliding was minimal, probably due to the lung-protective ventilator settings. B-lines were still present, obviously. In contrast to the normal clips, the pneumothorax clips were recorded with the transducer wrapped in a sterile cover. In theory, this might result in a slightly degraded US image.

Image quality might be overstated in video clips in which the diagnosis was made fast and perceived to be easy. Those clips might be scored good quality because they were 'easy' to assess.

A suggestion for future studies comparing ultrasound transducers might be to include subjects of all BMIs to better represent the general population of critically ill and injured patients.

\section{Conclusion}

In conclusion, we found no difference in the effectiveness of detecting or excluding a pneumothorax between a high-frequency linear-array ultrasound transducer, a curved-array, and a phased-array transducer. Besides many indications for which it is essential, the lineararray transducer produces the best image quality in lung ultrasonography. Based only on image quality, the linear-array transducer might qualify as the preferred transducer for lung ultrasonography and the preferred default in our prehospital setting.

\section{Abbreviations \\ CT: computed-tomography; IQR: interquartile range; HEMS: helicopter emergency medical service; NPV: negative predictive value; ONSD: optic nerve sheath diameter; PPV: positive predictive value; SD: standard deviation; TBI: traumatic brain injury; UGRA: ultrasound-guided regional anesthesia; US: ultrasonography; VATS: video-assisted thoracoscopic surgery.}

\section{Authors' contributions}

RK, EG, and GVG designed the study. RK, EG, and MK included the patients and assisted in data acquisition. RK, EG, and TR recorded the ultrasound video clips. RK analyzed the data. All authors contributed to writing and revising the manuscript. All authors read and approved the final manuscript.

\section{Author details \\ ${ }^{1}$ Department of Anesthesiology, Pain and Palliative Medicine, Radboud University Medical Center, Radboud Institute for Health Sciences, Geert Groot- eplein-Zuid 10, 6525 GA Nijmegen, The Netherlands. ${ }^{2}$ Helicopter Emergency Medical Service-Lifeliner 3, Radboud University Medical Center, Radboud Institute for Health Sciences, Geert Grooteplein-Zuid 10, 6525 GA Nijmegen, The Netherlands.}

\section{Acknowledgements}

We would like to thank the thoracic surgeons, scrub nurses, cardiothoracic anesthesiologists, nurse anesthetists, respiratory physicians, and respiratory nurse specialists of the Radboud university medical center for their invaluable assistance in the acquisition of the ultrasound clips.

We would like to thank Eva Cillessen for meticulously editing and cropping the ultrasound clips and Ewald Bronkhorst for his assistance with the statistical calculations. A sincere thank to all the HEMS physicians and expert anesthesiology residents (Loes Didden and Michiel van Lieshout) for their assessment of the video clips.

Many thanks to Roel van Vugt and Jouke Landman for their diligent proofreading of the manuscript.

\section{Competing interests}

The authors declare that they have no competing interests.

\section{Availability of data and materials}

The datasets used and analyzed during the current study are available from the corresponding author on reasonable request.

\section{Consent for publication}

Not applicable.

\section{Ethics approval and consent to participate}

Ethical approval was obtained from the institutional ethics review board of the Radboud university medical center, Nijmegen (file 2017-3487, chair: prof. Dekhuijzen, date of approval: 9 August 2017). Written informed consent was asked and obtained from every patient and from every observer.

Funding

Not applicable. 


\section{Publisher's Note}

Springer Nature remains neutral with regard to jurisdictional claims in published maps and institutional affiliations.

Received: 27 June 2018 Accepted: 11 September 2018

Published online: 22 October 2018

\section{References}

1. Chen SC, Markmann JF, Kauder DR, Schwab CW (1997) Hemopneumothorax missed by auscultation in penetrating chest injury. J Trauma 42(1):86-89

2. Kong VY, Sartorius B, Clarke DL (2015) The accuracy of physical examination in identifying significant pathologies in penetrating thoracic trauma. Eur J Trauma Emerg Surg 41(6):647-650

3. Hirshberg A, Thomson SR, Huizinga WK (1988) Reliability of physical examination in penetrating chest injuries. Injury 19(6):407-409

4. Rantanen NW (1986) Diseases of the thorax. Vet Clin North Am Equine Pract 2(1):49-66

5. Blaivas M, Lyon M, Duggal S (2005) A prospective comparison of supine chest radiography and bedside ultrasound for the diagnosis of traumatic pneumothorax. Acad Emerg Med 12(9):844-849

6. Nagarsheth K, Kurek S (2011) Ultrasound detection of pneumothorax compared with chest X-ray and computed tomography scan. Am Surg 77(4):480-484

7. Lichtenstein DA, Meziere GA (2008) Relevance of lung ultrasound in the diagnosis of acute respiratory failure: the BLUE protocol. Chest 134(1):117-125
8. Gillman LM, Kirkpatrick AW (2012) Portable bedside ultrasound: the visual stethoscope of the 21st century. Scand J Trauma Resusc Emerg Med 20:18

9. Wittenberg M (2014) Will ultrasound scanners replace the stethoscope? BMJ 348:93463

10. Price DD, Wilson SR, Murphy TG (2000) Trauma ultrasound feasibility during helicopter transport. Air Med J 19(4):144-146

11. Nelson BP, Melnick ER, Li J (2011) Portable ultrasound for remote environments, part I: feasibility of field deployment. J Emerg Med 40(2):190-197

12. Roline CE, Heegaard WG, Moore JC et al (2013) Feasibility of bedside thoracic ultrasound in the helicopter emergency medical services setting. Air Med J 32(3):153-157

13. Lichtenstein DA, Menu Y (1995) A bedside ultrasound sign ruling out pneumothorax in the critically ill: lung sliding. Chest 108(5):1345-1348

14. Lichtenstein DA (2014) Lung ultrasound in the critically ill. Ann Intensive Care 4(1):1

15. Adhikari S (2014) High-frequency transducers for point-of-care ultrasound applications: what is the optimal frequency range? Intern Emerg Med 9(4):463-466

16. Tasci O, Hatipoglu ON, Cagli B, Ermis V (2016) Sonography of the chest using linear-array versus sector transducers: correlation with auscultation, chest radiography, and computed tomography. J Clin Ultrasound 44(6):383-389

17. Rajajee V, Vanaman M, Fletcher JJ, Jacobs TL (2011) Optic nerve ultrasound for the detection of raised intracranial pressure. Neurocrit Care 15(3):506-515

18. Biegler N, McBeth PB, Tiruta C et al (2013) The feasibility of nurse practitioner-performed, telementored lung telesonography with remote physician guidance_-'a remote virtual mentor'. Crit Ultrasound J 5(1):5

\section{Submit your manuscript to a SpringerOpen ${ }^{\circ}$ journal and benefit from:}

- Convenient online submission

- Rigorous peer review

- Open access: articles freely available online

- High visibility within the field

- Retaining the copyright to your article

Submit your next manuscript at springeropen.com 